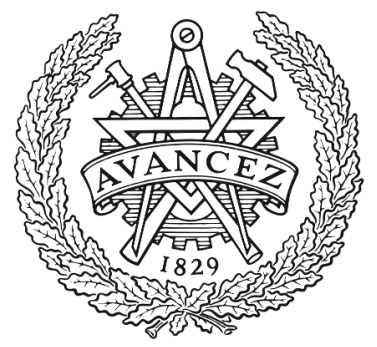

CHALMERS

UNIVERSITY OF TECHNOLOGY

\title{
5G Synchronization, Positioning, and Mapping from Diffuse Multipath
}

Downloaded from: https://research.chalmers.se, 2023-04-26 15:07 UTC

Citation for the original published paper (version of record):

Wen, F., Wymeersch, H. (2021). 5G Synchronization, Positioning, and Mapping from Diffuse Multipath. IEEE Wireless Communications Letters, 10(1): 43-47.

http://dx.doi.org/10.1109/LWC.2020.3020116

N.B. When citing this work, cite the original published paper.

(C)2021 IEEE. Personal use of this material is permitted.

However, permission to reprint/republish this material for advertising or promotional purposes 


\title{
5G Synchronization, Positioning, and Mapping from Diffuse Multipath
}

\author{
Fuxi Wen, Senior Member, IEEE and Henk Wymeersch, Senior Member, IEEE
}

\begin{abstract}
G mmWave communication systems have the potential to jointly estimate the positions of user equipment (UE) and mapping their propagation environments using a single base station. But such potential depends on the characteristics of the reflecting surfaces, such as a deterministic specular nature, a stochastic diffuse/scattering nature, or a combination of both. In this letter, we proposed a 5G positioning and mapping algorithm with unknown orientation and clock bias for single-bounce diffuse multipath channel models. The method is able to accurately localize, calibrate and synchronize the UE, even in the absence of line-of-sight and specular components. This enables robust positioning and mapping using only diffuse multipath.
\end{abstract}

Index Terms-5G mmWave, positioning and mapping, diffuse components, tensor decomposition, orientation and clock bias calibration

\section{INTRODUCTION}

5G mmWave communication systems provides new opportunities for improving the quality and robustness of communications, as well as for accurate user equipment (UE) positioning from a single base station (BS) [1]. Large bandwidth and multiple antennas, at both the BS and UE sides, allows for high resolution, with recent theoretical studies showing the potential of simultaneous positioning the UE and mapping the propagation environments utilizing the resolvable angleof-departures (AODs), angle-of-arrivals (AOAs), and time-ofarrivals (TOAs) [2], [3]. However, this potential depends on the characteristics of the objects in the environment, whereby propagation paths may be of a deterministic specular nature, or of a stochastic diffuse/scattering nature, or a combination of both. In general, as the frequency increases, surfaces appear rougher and lead to more diffuse scatterings [4], there is higher path loss, and higher sensitivity to line-of-sight (LOS) blockage. For non-LOS (NLOS) mmWave propagation, especially for vehicle-to-everything communications in urban scenarios [5], specular components together with single-bounce diffuse components form a majority of the overall channel gain [4]. Diffuse multipath is often treated as a disturbance, though geometry-based diffuse scattering channel models can also be considered, where each surface contributes with a cluster of paths, all with similar angles and delays [6], [7]. Such a model was exploited in the positioning and mapping problem [7]. However, [7] did not consider the impact of the UE's clock bias and orientation, both of which are important practical parameters. Joint localization and synchronization has been treated in several studies [8]-[12]: an analysis based on Fisher information theory in [8] showed that, under LOS, joint localization, synchronization and orientation estimation

F. Wen and H. Wymeersch are with the Department of Electrical Engineering, Chalmers University of Technology, Sweden. This work was supported, in part, by the Swedish Research Council under grant 2018-03701. are possible and that NLOS paths do not negatively impact the performance. [13] showed that NLOS paths from ideal reflections can be harness to improve performance, while [9] showed that the UE location and clock bias can be estimated with a sufficient number of NLOS paths, even when the LOS is blocked. In [10] a message passing method was proposed, which requires a priori knowledge of the position and clock bias. Furthermore, cooperative network synchronization [11] and single-anchor localization and synchronization of fullduplex agents [12] have also been proposed. Common among all these works is that only specular multipath is considered, while diffuse multipath is ignored.

In this letter, we extend [7] by using estimated diffuse and specular multipath parameters to recover the UE position, orientation, clock bias and the map of the propagation environment, for single-bounce channels. Our specific contributions are as follows:

- We propose a low-complexity processing chain (requiring only a 2D search) for estimating the UE position, orientation, clock bias, and the map of the propagation environment based on a single downlink transmission;

- We demonstrate that joint synchronization, positioning, and mapping is possible even when both LOS and specular multipath are absent.

Notation: Scalars are denoted in italic, e.g., $x$. Lower case boldface indicates a column vector, e.g., $\mathbf{x}$. Upper case boldface denotes a matrix, e.g., $\mathbf{X}$ with $\mathbf{I}_{N}$ representing an $N \times N$ identity matrix, while upper case boldface calligraphic denotes a tensor, e.g., $\mathcal{X}$. Matrix transpose, conjugate transpose, and inverse are indicated by superscript ${ }^{\top}, \mathrm{H}^{\mathrm{a}}$ and ${ }^{-1}$, respectively. The Euclidean norm is denoted by $\|\cdot\|$, while the tensor Frobenius norm is indicated by $\|\cdot\|_{F}$. The expectation operator is denoted by $\mathbb{E}\{\cdot\}$ and the set of all complex numbers is denoted by $\mathbb{C}$.

\section{System Model And Problem Formulation}

We consider a 3D scenario with a single transmitting $\mathrm{BS}$ with known location $\boldsymbol{p}_{\mathrm{T}}=\left[x_{\mathrm{T}}, y_{\mathrm{T}}, z_{\mathrm{T}}\right]^{\top}$ and a receiving UE with unknown location $\boldsymbol{p}_{\mathrm{R}}=\left[x_{\mathrm{R}}, y_{\mathrm{R}}, z_{\mathrm{R}}\right]^{\top}$, as well as an unknown clock bias expressed in meters $\beta c$ ( $c$ is the speed of light) and an unknown orientation $\alpha \in[0,2 \pi)$ with respect to the vertical axis. There are $R$ reflectors and each reflector gives rise to $N_{r} \geq 1$ rays [14], with the $l$-th ray corresponding to an unknown and random incidence point (a scattering point (SP)) on the $r$ th reflecting surface in the environment, denoted by $\boldsymbol{p}_{r l}=\left[x_{r l}, y_{r l}, z_{r l}\right]^{\mathrm{\top}}$, whose distribution on the surface depends on the electromagnetic properties [15]. The LOS path, if it exists, has index $r=0$, with $N_{0}=1$. 
The BS and UE both employ uniform rectangular arrays (URAs) consist of sensors in a grid of size $M_{\mathrm{T}}=M_{1} \times M_{2}$ and $M_{\mathrm{R}}=M_{3} \times M_{4}$, and transmit MIMO-OFDM signals (other signals may be used provided they are compatible with the signal model (3), to be defined later) with $M_{5}$ subcarriers and sub-carrier spacing $\Delta_{f}$. The channel parameters associated with incidence point $\boldsymbol{p}_{r l}$ are the 6-tuple of (i) AOD in azimuth and elevation angles $\left(\phi_{r l}, \psi_{r l}\right)$ at the BS; (ii) AOA in azimuth and elevation angles $\left(\theta_{r l}, \varphi_{r l}\right)$ at UE; (iii) the TOA $\tau_{r l}$; and (v) the complex channel gain $\gamma_{r l}$. The models for the channel gains follows the standard path loss model for the LOS path and specular components, while for diffuse components, each gain has identical magnitude and uniform phase [7].

The downlink frequency-domain channel response for subcarrier $f_{i}$ is represented as $\mathbf{H}_{i} \in \mathbb{C}^{M_{R} \times M_{T}}$ is expressed as

$$
\mathbf{H}_{i}=\sum_{r=0}^{R} \sum_{l=1}^{N_{r}} \gamma_{r l} e^{-j 2 \pi f_{i} \tau_{r l}} \mathbf{a}_{\mathrm{R}}\left(\theta_{r l}, \varphi_{r l}\right) \mathbf{a}_{\mathrm{T}}^{\mathrm{H}}\left(\phi_{r l}, \psi_{r l}\right),
$$

where $\mathbf{a}_{\mathrm{R}}\left(\theta_{r l}, \varphi_{r l}\right)$ and $\mathbf{a}_{\mathrm{T}}\left(\phi_{r l}, \psi_{r l}\right)$ are the antenna array response vectors at the UE and BS, respectively [6], [16]. For subcarrier $i, \mathbf{H}_{i}$ can be converted in a $4 \mathrm{D}$ tensor of suitable dimension, $\mathcal{H}_{i} \in \mathbb{C}^{M_{1} \times M_{2} \times M_{3} \times M_{4}}$ [17]. The received signal on subcarrier $i$ is of the form $\mathbf{Y}_{i}=\mathbf{H}_{i} \mathbf{S}_{i}+\mathbf{N}_{i}$, where $\mathbf{S}_{i}$ is a known pilot signal with orthogonality property $\left(\mathbf{S}_{i} \mathbf{S}_{i}^{\mathrm{H}}=\right.$ $\rho \mathbf{I}_{M_{T}}$ ) and $\mathbf{N}_{i}$ is i.i.d. Gaussian noise. Then we have $\mathbf{X}_{i}=$ $\frac{1}{\rho} \mathbf{Y}_{i} \mathbf{S}_{i}^{\mathrm{H}}=\mathbf{H}_{i}+\frac{1}{\rho} \mathbf{N}_{i} \mathbf{S}_{i}^{\mathrm{H}}=\mathbf{H}_{i}+\mathbf{W}_{i}$, where $\mathbf{W}_{i}$ is also i.i.d. Gaussian noise with a scaled covariance matrix.

Our objective is, given measurements $\mathbf{X}_{i}$ of the mmWave channel, to determine $\boldsymbol{p}_{\mathrm{UE}}, B, \alpha$ and a parametrization of the $K$ surfaces. This involves three steps: (i) estimation and association of parameters of specular and diffuse multipath, (ii) localization, synchronization, and orientation estimation; (iii) mapping.

\section{Proposed Method}

\section{A. Estimation and Association of Specular and Diffuse Mul- tipath Parameters}

Remark 1. While there are many mmWave channel estimation methods, our application has two specific requirements. First of all, for each estimated path, the AOA, AOD, and TOA should be correctly matched. In other words, the channel estimator should return 5D tuples, not unordered sets of angles and delays. Secondly, the estimator should provide such tuples not only for the specular paths, but also for diffuse components. This is in contrast to the conventional approach, which treats diffuse multipath as a random process without exploiting its relation to the environment geometry. To the best of our knowledge, tensor-ESPRIT (estimation of signal parameters via rotational invariance techniques) [18] is unique in meeting these two requirements and explained briefly here.

We exploit the $R$-D grid structure inherent in the data, as well as the Vandermonde structure in angle and delay domain to map from geometric channel parameters to spatial frequencies. For subcarrier $i, \mathbf{X}_{i}$ and $\mathbf{W}_{i}$ are $M_{3} M_{4} \times M_{1} M_{2}$ matrices. We convert these $M_{5}$ matrices (one per subcarrier) in a $5 \mathrm{D}$ tensor of suitable dimension, $\mathcal{X}, \mathcal{H}$ and $\mathcal{W} \in$ $\mathbb{C}^{M_{1} \times M_{2} \times M_{3} \times M_{4} \times M_{5}}$. For the $p$ th path $(p=1,2, \cdots, P$ where $P=\sum_{k=1}^{R} N_{k}$, where we have renumbered the paths compared to (1)) and $d$-th dimension $(d \in\{1,2, \cdots, 5\})$, we now introduce $\mathbf{a}\left(\omega_{p}^{(d)}\right) \in \mathbb{C}^{M_{d} \times 1}$, which is equivalent to the uniform linear array steering vector composed of $M_{d}$ sensors, and $\omega_{p}^{(d)}$ is the spatial frequency, with [17]

$$
\begin{aligned}
& \omega_{p}^{(1)}=\pi \sin \left(\phi_{p}\right) \cos \left(\psi_{p}\right), \quad \omega_{p}^{(2)}=\pi \sin \left(\psi_{p}\right), \\
& \omega_{p}^{(3)}=\pi \sin \left(\theta_{p}\right) \cos \left(\varphi_{p}\right), \quad \omega_{p}^{(4)}=\pi \sin \left(\varphi_{p}\right), \\
& \omega_{p}^{(5)}=2 \pi \Delta_{f} \tau_{p} .
\end{aligned}
$$

For the $p$ th path, the equivalent $5 \mathrm{D}$ array steering tensor can be written as $\mathcal{A}_{p}=\mathbf{a}\left(\omega_{p}^{(1)}\right) \circ \mathbf{a}\left(\omega_{p}^{(2)}\right) \circ \cdots \circ \mathbf{a}\left(\omega_{p}^{(5)}\right)$, where $\circ$ represents the outer product. This allows us to express the observation as

$$
\mathcal{X}=\sum_{p=1}^{P} \gamma_{p} \mathcal{A}_{p}+\mathcal{W} \in \mathbb{C}^{M_{1} \times M_{2} \times \cdots \times M_{5}}
$$

This formulation directly allows us to apply tensor-ESPRIT [18] for channel estimation with high resolution and limited computational complexity. The basic idea of tensor-ESPRIT is exploiting the multidimensional shift invariant structure of $\mathcal{A}_{p}$. Here, the spatial frequency estimates are obtained without nonlinear optimization and without computing or searching of any spatial spectrum. The number of signal components is a prerequisite for subspace based methods, such as tensorESPRIT. In the the CP decomposition model [19], tensor $\mathcal{X}$ is decomposed into a sum of rank-one tensors, each component corresponds to one path. Model order selection techniques [20] can be used to detect the number of paths $\hat{P}$. In general, the estimated $\hat{P} \ll P$ for rough surfaces with hundreds of closely located diffuse paths. The reason the number of paths is underestimated is due to the finite resolution of the receiver. The diffuse multipath is generated from a very large number of scatter points on the surface of each object, densely spaced to approximate the underlying continuous distribution. The receiver can only resolve paths to the extent allowed by its bandwidth (delay resolution) and array size (angular resolution). Finally, the estimated spatial frequencies $\left\{\hat{\omega}_{k}^{(d)}\right\}$ for $d \in\{1,2, \cdots, 5\}$ and $k \in\{1,2, \cdots, \hat{P}\}$ are converted to AOA, AOD and TOA, via (2). Additional details can be found in [7]. Each of the $\hat{P}$ estimated paths can be associated with a 3D point on one of the $R$ surfaces. We denote these points as artificial SPs, since they will be be different from the $P$ physical SPs $\boldsymbol{p}_{r l}$ corresponding to the rays. Both the physical SPs and the artificial SPs are random. However, the proposed method does not require knowledge of their distributions.

\section{B. Positioning, Synchronization, and Orientation Estimation}

We make use of the following essential geometric relations. For the LOS path, we introduce $\boldsymbol{p}_{01}$ as any point on the line strictly between $\boldsymbol{p}_{\mathrm{T}}$ and $\boldsymbol{p}_{\mathrm{R}}$, but not equal to either point. Then the following relations hold for any path, i.e., $\forall r, l$ :

$$
\begin{aligned}
\tau_{r l} & =\left\|\boldsymbol{p}_{r l}-\boldsymbol{p}_{\mathrm{T}}\right\| / c+\left\|\boldsymbol{p}_{r l}-\boldsymbol{p}_{\mathrm{R}}\right\| / c+\beta \\
\theta_{r l} & =\pi+\operatorname{atan} 2\left(y_{r l}-y_{\mathrm{R}}, x_{r l}-x_{\mathrm{R}}\right)-\alpha \\
\varphi_{r l} & =\operatorname{asin}\left(z_{r l}-z_{\mathrm{R}}\right) /\left\|\boldsymbol{p}_{r l}-\boldsymbol{p}_{\mathrm{R}}\right\| \\
\psi_{r l} & =\operatorname{asin}\left(z_{r l}-z_{\mathrm{T}}\right) /\left\|\boldsymbol{p}_{r l}-\boldsymbol{p}_{\mathrm{T}}\right\| \\
\phi_{r l} & =\operatorname{atan} 2\left(y_{r l}-y_{\mathrm{T}}, x_{r l}-x_{\mathrm{T}}\right) .
\end{aligned}
$$


Due to the presence of $\alpha$ and $\beta$, solving for the UE location is not trivial. We propose a two-step approach. We first determine an estimate of the UE location, assuming known orientation and clock bias. This estimate is denoted by $\hat{\boldsymbol{p}}_{\mathrm{R}}(\alpha, \beta)$. Then we optimize over all pairs $(\alpha, \beta)$ of orientations and clock biases.

1) Estimation of $\hat{\boldsymbol{p}}_{\mathrm{R}}(\alpha, \beta)$ : The estimation problem is challenging, due to the lack of knowledge on whether the LOS path is present. We tackle the problem based on the methods from [21], [22]. We first define

$$
\boldsymbol{f}_{\mathrm{T}, k}=\left[\begin{array}{c}
\cos \left(\hat{\phi}_{k}\right) \cos \left(\hat{\theta}_{k}\right) \\
\cos \left(\hat{\phi}_{k}\right) \sin \left(\hat{\theta}_{k}\right) \\
\sin \left(\hat{\phi}_{k}\right)
\end{array}\right]
$$

which points along the AOD of path $k \in\{1, \ldots, \hat{P}\}$; and $\boldsymbol{f}_{\mathrm{R}, \mathrm{k}}$ is defined equivalently for the AOA, pointing from the UE towards the $k$-th artificial SP:

$$
\boldsymbol{f}_{\mathrm{R}, k}=\left[\begin{array}{c}
\cos \left(\hat{\phi}_{k}\right) \cos \left(\hat{\theta}_{k}+\alpha-\pi\right) \\
\cos \left(\hat{\phi}_{k}\right) \sin \left(\hat{\theta}_{k}+\alpha-\pi\right) \\
\sin \left(-\hat{\phi}_{k}\right)
\end{array}\right],
$$

where we recall that $\alpha$ is assumed known in this first step.

For each path $k$, we can establish the following relation:

$$
\boldsymbol{p}_{\mathrm{R}}=\boldsymbol{p}_{\mathrm{T}}+c \tilde{\tau}_{k} \xi_{k} \boldsymbol{f}_{\mathrm{T}, k}+c \tilde{\tau}_{k}\left(1-\xi_{k}\right)\left(-\boldsymbol{f}_{\mathrm{R}, k}\right),
$$

where $\tilde{\tau}_{k}=\hat{\tau}_{k}-\beta$, where $\xi_{k} \in[0,1]$ is unknown and represents the fraction of the delay $\tilde{\tau}_{k}$ that is attributed to the line from BS to a scatter point. Note that the value of $\xi_{k}$ is arbitrary for the LOS path (if it is present). Rearranging the terms in (7) results in

$$
\boldsymbol{p}_{\mathrm{R}}=\boldsymbol{\delta}_{k}+\xi_{k} \boldsymbol{u}_{k}, \quad k \in\{1, \ldots, \hat{P}\},
$$

with $\boldsymbol{\delta}_{k}=\boldsymbol{p}_{\mathrm{T}}-c \tilde{\tau}_{k} \boldsymbol{f}_{\mathrm{R}, k}$ and $\boldsymbol{u}_{k}=c \tilde{\tau}_{k}\left(\boldsymbol{f}_{\mathrm{T}, k}+\boldsymbol{f}_{\mathrm{R}, k}\right)$. From (8), we see that $\xi_{k}\left\|\boldsymbol{u}_{k}\right\|^{2}=\boldsymbol{u}_{k}^{\top}\left(\boldsymbol{p}_{\mathrm{R}}-\boldsymbol{\delta}_{k}\right)$. Solving for $\xi_{k}$ and substituting back into (8) provides us with the following cost function

$$
\mathcal{C}\left(\boldsymbol{p}_{\mathrm{R}}\right)=\sum_{k=1}^{\hat{P}} \zeta_{k}\left\|\boldsymbol{p}_{\mathrm{R}}-\left(\boldsymbol{\delta}_{k}+\overline{\boldsymbol{u}}_{k}^{\top}\left(\boldsymbol{p}_{\mathrm{R}}-\boldsymbol{\delta}_{k}\right) \overline{\boldsymbol{u}}_{k}\right)\right\|^{2}
$$

where $\overline{\boldsymbol{u}}_{k}=\boldsymbol{u}_{k} /\left\|\boldsymbol{u}_{k}\right\|$ and $\zeta_{k} \geq 0$ is the weight of the $k$-th path, which can depend on the channel gain (amplitude) of the path. Minimizing $\mathcal{C}\left(\boldsymbol{p}_{\mathrm{R}}\right)$ yields a closed-form solution

$$
\hat{\boldsymbol{p}}_{\mathrm{R}}(\alpha, \beta)=\left(\sum_{k=1}^{\hat{P}} \zeta_{k}\left(\boldsymbol{I}-\overline{\boldsymbol{u}}_{k} \overline{\boldsymbol{u}}_{k}^{\top}\right)\right)^{-1} \sum_{k=1}^{\hat{P}} \zeta_{k}\left(\boldsymbol{I}-\overline{\boldsymbol{u}}_{k} \overline{\boldsymbol{u}}_{k}^{\top}\right) \boldsymbol{\delta}_{k} .
$$

Note that the method does not require separation of specular and diffuse paths, nor does it rely on knowledge of which path is the LOS (or even presence of the LOS). The cost function in (9) can be applied with all $\hat{P}$ estimated paths, or only a selected subset of paths (e.g., the strongest ones).

2) Estimation of $(\alpha, \beta)$ : Substituting $\hat{\boldsymbol{p}}_{\mathrm{R}}(\alpha, \beta)$ back into (9) yields a cost function over $(\alpha, \beta)$, i.e., $\mathcal{C}(\alpha, \beta)=$ $\mathcal{C}\left(\hat{\boldsymbol{p}}_{\mathrm{R}}(\alpha, \beta)\right)$, (without depending on the unknown $\left.\boldsymbol{p}_{\mathrm{R}}\right)$ from which $(\alpha, \beta)$ can be found by solving

$$
\begin{aligned}
\operatorname{minimize} & \mathcal{C}(\alpha, \beta) \\
\text { s.t. } & \alpha \in\left[\alpha_{\min },+\alpha_{\max }\right], \\
& \beta \in\left[\beta_{\min },+\beta_{\max }\right],
\end{aligned}
$$

with a 2D grid search. Geometric constraints can be introduced to limit the size of the search space [23]. Denoting the solution to $(11)$ by $(\hat{\alpha}, \hat{\beta})$, the final estimate of the UE location is $\hat{\boldsymbol{p}}_{\mathrm{R}}(\hat{\alpha}, \hat{\beta})$.

Remark 2 (Intuition of the method). The UE state can be estimated by noticing that each estimated artificial SP provides 5 observations (1 delay and 4 angles), while being parameterized by 3 unknowns (the artificial SP position). Hence, the system is over-determined, so that with a sufficient number of estimated paths, all unknowns can be estimated, even if the artificial SP locations are unknown. Note that in contrast to [9], a single sufficiently rough surface is sufficient to provide an unambiguous UE state estimate in the absence of LOS.

\section{Mapping the Propagation Environment}

Finally, we use the estimates of the UE state to determine estimates of the environment. Given $\hat{\boldsymbol{p}}_{\mathrm{R}}(\hat{\alpha}, \hat{\beta}), \hat{\alpha}$ and $\hat{\beta}$, we can recover artificial SPs $\boldsymbol{p}_{k}, k \in\{1, \ldots, \hat{P}\}$ as the intersection of the lines $\boldsymbol{p}_{\mathrm{T}}+\kappa_{\mathrm{T}} \boldsymbol{f}_{\mathrm{T}, k}, \kappa_{\mathrm{T}} \in \mathbb{R}$ and $\boldsymbol{p}_{\mathrm{R}}+\kappa_{\mathrm{R}} \boldsymbol{f}_{\mathrm{R}, k}, \kappa_{\mathrm{R}} \in \mathbb{R}$. The least-squares solution is readily found to be

$$
\hat{\boldsymbol{p}}_{k}=\left(\mathbf{H}_{\mathrm{T}, k}+\mathbf{H}_{\mathrm{R}, k}\right)^{-1}\left(\mathbf{H}_{\mathrm{T}, k} \boldsymbol{p}_{\mathrm{T}}+\mathbf{H}_{\mathrm{R}, k} \hat{\boldsymbol{p}}_{\mathrm{R}}(\hat{\alpha}, \hat{\beta})\right),
$$

with $\mathbf{H}_{\mathrm{T}, k}=\mathbf{I}-\boldsymbol{f}_{\mathrm{T}, k} \boldsymbol{f}_{\mathrm{T}, k}^{\top}, \mathbf{H}_{\mathrm{R}, k}=\mathbf{I}-\boldsymbol{f}_{\mathrm{R}, k} \boldsymbol{f}_{\mathrm{R}, k}^{\top}$. Note that $\hat{\boldsymbol{p}}_{k}$ need not correspond to any physical SP $\boldsymbol{p}_{r l}$, due to the limited resolution in angle and delay of the $5 \mathrm{G}$ mmWave communication system.

In order to attach a meaning to the artificial SPs, a post-processing step is needed. The artificial SPs are first partitioned into clusters, say $\mathcal{C}_{1}, \mathcal{C}_{2}, \cdots, \mathcal{C}_{\hat{K}}$ using standard clustering methods. Each cluster $\mathcal{C}_{c}$ ideally corresponds to one surface and can then be represented by a mean $\boldsymbol{\mu}_{c}$ and a covariance $\boldsymbol{\Sigma}_{c}$, given by

$$
\begin{aligned}
\boldsymbol{\mu}_{c} & =\frac{1}{\left|\mathcal{C}_{c}\right|} \sum_{i \in \mathcal{C}_{c}} \boldsymbol{p}_{i}, \\
\boldsymbol{\Sigma}_{c} & =\frac{1}{\left|\mathcal{C}_{c}\right|-1} \sum_{i \in \mathcal{C}_{c}}\left(\boldsymbol{p}_{i}-\boldsymbol{\mu}_{c}\right)\left(\boldsymbol{p}_{i}-\boldsymbol{\mu}_{c}\right)^{\top}
\end{aligned}
$$

where $|\cdot|$ denotes the number of elements of the set. The proposed algorithm is summarized in Algorithm 1. Note that equations (5), (7)-(10) for positioning and (12) for mapping are the same as in [7].

\section{NuMERICAL RESUlTS}

\section{A. Simulation Setup}

We consider a carrier frequency of $28 \mathrm{GHz}$, corresponding to $\lambda=1.07 \mathrm{~cm}$, a total bandwidth of $20 \mathrm{MHz}$ with 100 subcarriers, of which 10 equally spaced subcarriers are used for pilots. A cyclic prefix of length 7 is used. 64 pilot OFDM symbols are sent, for a total duration of $3.52 \mathrm{~ms}$. We set the pilots as $\mathbf{S}_{i}=\mathbf{I}_{M_{T}}, \forall i$. The $\mathrm{BS}$ and UE are located at $\boldsymbol{p}_{\mathrm{T}}=[20,0,8]^{\top}$ and $\boldsymbol{p}_{\mathrm{R}}=[0,0,2]^{\top}$ with unknown orientation $\alpha=\pi / 3$ and clock bias $\beta c=4 \mathrm{~m}$, respectively. There two reflecting surfaces: one building facade and a ground surface. The $10 \mathrm{~m}$ high and $20 \mathrm{~m}$ long building facade's center is at $[10,10,5]^{\top}$, with orientation $[0,1,0]^{\top}(x-z$ plane $)$. 


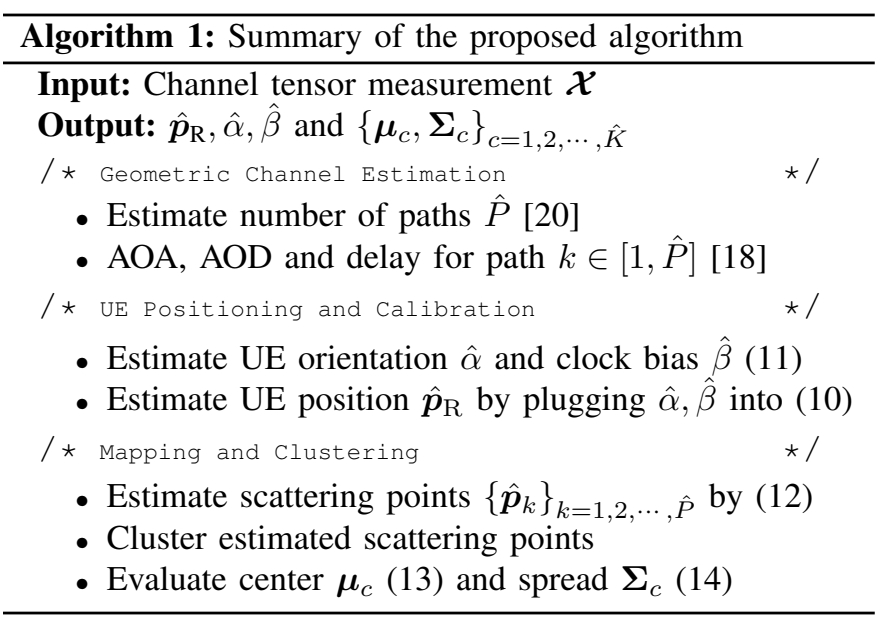

The second surface is at $[10,0,0]^{\top}$ with orientation $[0,0,1]^{\top}$ (reflected from ground, $x-y$ plane), surface dimension is $20 \mathrm{~m}$ $\times 20 \mathrm{~m}$. Both surfaces are described as rough surfaces with one specular component each, using $N_{r}=50$ scatter points each. Directivity parameter $\alpha_{R}=10$ and scattering coefficient $S=0.6$ are used to describe the roughness of the surface. The BS and UE are equipped with URAs with $(8 \times 8)$ elements and placed along $y-z$ plane. The inter-element spacing is $0.5 \lambda$ in both directions. and the origin is the array reference point. The Matlab package Tensorlab [24] is utilized for tensor computation.

\section{B. Results}

We assess the capability of the proposed algorithm to estimate the positioning, clock bias and orientation using the root-mean-square error $\left(\mathrm{RMSE}^{1}\right)$, defined for the position as $\mathrm{RMSE}=\sqrt{\mathbb{E}\left\{\left\|\boldsymbol{p}_{\mathrm{R}}-\hat{\boldsymbol{p}}_{\mathrm{R}}(\hat{\alpha}, \hat{\beta})\right\|^{2}\right\}}$, and similarly for the bias and orientation, for different levels of signal-to-noise ratio (SNR), which is defined as $\mathrm{SNR}=\|\mathcal{X}-\mathcal{N}\|_{F}^{2} /\|\mathcal{N}\|_{F}^{2}$. We consider four different combinations ${ }^{2}$ of utilizing the LOS path, specular components (interpreted as the shortest estimated path for each cluster) and diffuse paths, given by:

C1. LOS plus the two specular components (so that $N_{0}=1$, $\left.N_{1}=1, N_{2}=1\right)$.

C2. LOS plus the diffuse paths for each cluster (so that $N_{0}=$ $1, N_{1} \gg 1, N_{2} \gg 1$, no specular path).

C3. The specular components plus the diffuse paths (so that $N_{0}=0, N_{1} \gg 1, N_{2} \gg 1$ )

C4. Only the diffuse paths for each cluster (so that $N_{0}=0$, $N_{1} \gg 1, N_{2} \gg 1$, no specular path).

Note that the definition of the SNR implies that the effective transmit power in $\mathrm{C} 4$ will be larger than $\mathrm{C} 3$, which in turn will be larger than $\mathrm{C} 1$ and $\mathrm{C} 2$.

1) Positioning: Fig. 1 shows the positioning performance of the four combination. We also show two benchmarks: B1. without estimating the orientation and clock bias (without

\footnotetext{
${ }^{1}$ Since the diffuse paths cannot be resolved and the artificial SPs are a sideproduct of the channel estimation method itself, standard Cramér-Rao bounds considering separated sources cannot be applied.

${ }^{2}$ Note that some combinations will have low signal power $\|\mathcal{X}\|_{F}^{2}$, so that for a fixed SNR, the noise power $\|\mathcal{N}\|_{F}^{2}$ will also be low.
}

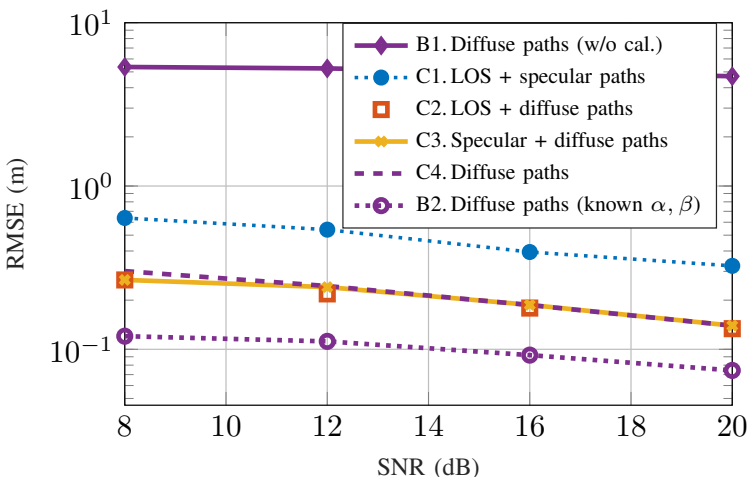

Fig. 1. UE positioning: RMSE versus SNR for different combinations of availability of the LOS path and specular components.

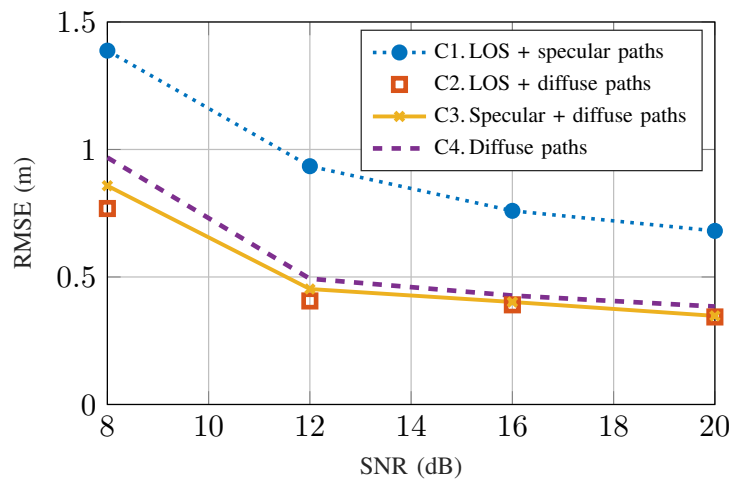

Fig. 2. UE clock bias estimation: RMSE versus SNR for different combinations of availability of the LOS path and specular components.

calibration, meaning that the UE assumes $(\alpha, \beta)=(0,0))$ and B2. with known orientation and clock bias $(\alpha, \beta)$. All variations use weights $\zeta_{k}=1$. The largest positioning error occurs for benchmark algorithm B1. That is because the incorrect orientation $\alpha$ and clock bias $\beta$ is assumed in (10). Sub-meter accuracy is achievable by using LOS and two specular paths. The smallest positioning error occurs for B2, indicating the importance of knowledge of the clock bias and orientation. Among the four combinations $\mathrm{C} 1-\mathrm{C} 4$, the worst performance is obtained when the diffuse paths are ignored (C1). The combinations $\mathrm{C} 2-\mathrm{C} 4$ all yield similar RMSE, with $\mathrm{C} 2$ providing the best performance. Giving a larger weight to LOS and specular paths in (9) can provide additional gains, but was not explored in this letter.

2) Synchronization and orientation estimation: Fig. 2 shows the corresponding results for the clock bias (without the benchmarks, which are meaningless here), which largely follows the positioning performance. In terms of orientation estimation, Fig. 3, lower RMSE is achieved by utilizing the resolvable diffuse paths. Slightly improvements are observable by including the LOS and specular paths.

3) Mapping: Mapping performance at $20 \mathrm{~dB}$ SNR in terms of the projection onto the $x-y$ and $x-z$ planes is shown in Fig. 4. We observe relatively good performance for both surfaces. Both the location and shape of the surfaces can be recovered. 


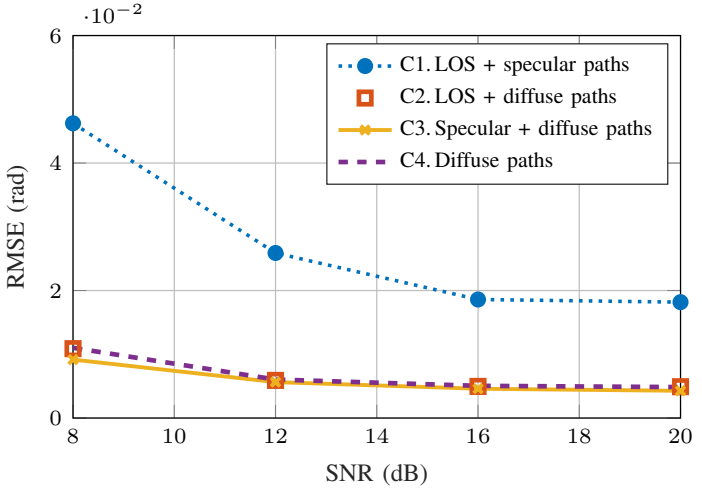

Fig. 3. UE orientation estimation: RMSE versus SNR for different combinations of availability of the LOS path and specular components.
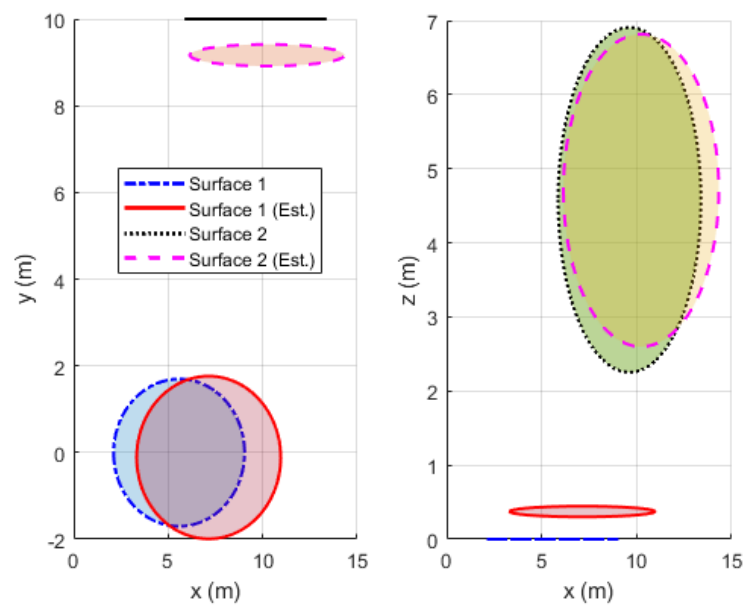

Fig. 4. Mapping results (projection onto the $x-y$ and $x-z$ planes), SNR $=$ $20 \mathrm{~dB}$, LOS is present, clock bias $\beta c=4 \mathrm{~m}$ and orientation $\alpha=\pi / 3$ are unknown.

\section{CONCLUSIONS}

We studied the problem of geometric parameter estimation in mmWave MIMO communications with diffuse scattering components, combined with UE orientation and clock bias estimation. We proposed a positioning and mapping method based on the estimated geometric channel parameters, and demonstrate that accurate UE positioning and propagation environment mapping is possible, even when the LOS path is blocked and specular components are absent, the reflecting surfaces are only characterized by diffuse scattering. Moreover, the method does not require identifying LOS or specular signal components. Modifications are needed for multi-bounce scenarios.

\section{REFERENCES}

[1] R. Mendrzik, F. Meyer, G. Bauch, and M. Z. Win, "Enabling situational awareness in millimeter wave massive MIMO systems," IEEE Journal of Selected Topics in Signal Processing, vol. 13, no. 5, pp. 1196-1211, 2019.

[2] A. Shahmansoori, G. E. Garcia, G. Destino, G. Seco-Granados, and $\mathrm{H}$. Wymeersch, "Position and orientation estimation through millimeterwave MIMO in 5G systems," IEEE Transactions on Wireless Communications, vol. 17, no. 3, pp. 1822-1835, 2018.
[3] R. Mendrzik, H. Wymeersch, G. Bauch, and Z. Abu-Shaban, "Harnessing NLOS components for position and orientation estimation in $5 \mathrm{G}$ millimeter wave MIMO," IEEE Transactions on Wireless Communications, vol. 18, no. 1, pp. 93-107, 2019.

[4] C. Wang, J. Bian, J. Sun, W. Zhang, and M. Zhang, "A survey of 5G channel measurements and models," IEEE Communications Surveys Tutorials, vol. 20, no. 4, pp. 3142-3168, 2018.

[5] B. Antonescu, M. T. Moayyed, and S. Basagni, "Diffuse scattering models for mmwave V2X communications in urban scenarios," in International Conference on Computing, Networking and Communications (ICNC), 2019, pp. 923-929.

[6] N. Czink, F. Kaltenberger, Y. Zhou, L. Bernadó, T. Zemen, and X. Yin, "Low-complexity geometry-based modeling of diffuse scattering," in Proceedings of the Fourth European Conference on Antennas and Propagation, 2010, pp. 1-4.

[7] F. Wen, J. Kulmer, K. Witrisal, and H. Wymeersch, "5G positioning and mapping with diffuse multipath," arXiv preprint arXiv:1912.08697, 2020.

[8] A. Guerra, F. Guidi, and D. Dardari, "Single-anchor localization and orientation performance limits using massive arrays: MIMO vs. beamforming," IEEE Transactions on Wireless Communications, vol. 17, no. 8, pp. 5241-5255, 2018.

[9] H. Wymeersch, N. Garcia, H. Kim, G. Seco-Granados, S. Kim, F. Wen, and M. Fröhle, "5G mm wave downlink vehicular positioning," in 2018 IEEE Global Communications Conference (GLOBECOM), 2018, pp. 206-212.

[10] R. Mendrzik, F. Meyer, G. Bauch, and M. Win, "Localization, mapping, and synchronization in 5G millimeter wave massive MIMO systems," in International Workshop on Signal Processing Advances in Wireless Communications (SPAWC). IEEE, 2019, pp. 1-5.

[11] Y. Xiong, N. Wu, Y. Shen, and M. Z. Win, "Cooperative network synchronization: Asymptotic analysis," IEEE Transactions on Signal Processing, vol. 66, no. 3, pp. 757-772, 2017.

[12] Y. Liu, Y. Shen, and M. Z. Win, "Single-anchor localization and synchronization of full-duplex agents," IEEE Transactions on Communications, vol. 67, no. 3, pp. 2355-2367, 2018.

[13] Y. Wang, Y. Wu, and Y. Shen, "Joint spatiotemporal multipath mitigation in large-scale array localization," IEEE Transactions on Signal Processing, vol. 67, no. 3, pp. 783-797, 2018.

[14] R. He, B. Ai, A. F. Molisch, G. L. Stuber, Q. Li, Z. Zhong, and J. Yu, "Clustering enabled wireless channel modeling using big data algorithms," IEEE Communications Magazine, vol. 56, no. 5, pp. $177-$ $183,2018$.

[15] J. Kulmer, "High-accuracy positioning exploiting multipath for reducing the infrastructure," Ph.D. dissertation, Graz University of Technology (90000), 32019.

[16] C. F. Mecklenbrauker, A. F. Molisch, J. Karedal, F. Tufvesson, A. Paier, L. Bernado, T. Zemen, O. Klemp, and N. Czink, "Vehicular channel characterization and its implications for wireless system design and performance," Proceedings of the IEEE, vol. 99, no. 7, pp. 1189-1212, 2011.

[17] P. Heidenreich, A. M. Zoubir, and M. Rubsamen, "Joint 2-D DOA estimation and phase calibration for uniform rectangular arrays," IEEE Transactions on Signal Processing, vol. 60, no. 9, pp. 4683-4693, 2012.

[18] M. Haardt, F. Roemer, and G. Del Galdo, "Higher-order SVD-based subspace estimation to improve the parameter estimation accuracy in multidimensional harmonic retrieval problems," IEEE Transactions on Signal Processing, vol. 56, no. 7, pp. 3198-3213, 2008.

[19] T. G. Kolda and B. W. Bader, "Tensor decompositions and applications," SIAM Review, vol. 51, no. 3, pp. 455-500, Aug. 2009.

[20] K. Liu, J. P. C. da Costa, H. C. So, L. Huang, and J. Ye, "Detection of number of components in CANDECOMP/PARAFAC models via minimum description length," Digital Signal Processing, vol. 51, pp. $110-123,2016$

[21] H. Wymeersch, "A simple method for $5 \mathrm{G}$ positioning and synchronization without line-of-sight," arXiv preprint arXiv:1812.05417, 2018.

[22] F. Wen, H. C. So, and H. Wymeersch, "Tensor decomposition-based beamspace ESPRIT algorithm for multidimensional harmonic retrieval," in IEEE International Conference on Acoustics, Speech and Signal Processing (ICASSP), 2020, pp. 4572-4576.

[23] R. Mendrzik, F. Meyer, G. Bauch, and M. Win, "Fast inference for situational awareness in 5G millimeter wave massive MIMO systems," in 2019 IEEE 20th International Workshop on Signal Processing Advances in Wireless Communications (SPAWC), 2019, pp. 1-5.

[24] N. Vervliet, O. Debals, L. Sorber, M. Van Barel, and L. De Lathauwer. (2016, Mar.) Tensorlab 3.0. [Online]. Available: https://www.tensorlab.net 